Review Article

\title{
Danhong Injection (a Traditional Chinese Patent Medicine) for Acute Myocardial Infarction: A Systematic Review and Meta-Analysis
}

\author{
Pengda Liao, ${ }^{1,2}$ Lei Wang, ${ }^{1,2}$ Liheng Guo, ${ }^{1,2}$ Ruixiang Zeng, \\ Juming Huang, ${ }^{1,2}$ and Minzhou Zhang ${ }^{1,2}$ \\ ${ }^{1}$ Guangdong Provincial Hospital of Chinese Medicine, Guangzhou 510120, China \\ ${ }^{2}$ The 2nd Clinical College of Guangzhou University of Chinese Medicine, Guangzhou 510405, China
}

Correspondence should be addressed to Minzhou Zhang; minzhouzhang@aliyun.com

Received 19 May 2015; Accepted 17 August 2015

Academic Editor: Kristine McGrath

Copyright (c) 2015 Pengda Liao et al. This is an open access article distributed under the Creative Commons Attribution License, which permits unrestricted use, distribution, and reproduction in any medium, provided the original work is properly cited.

Objective. We aimed to systematically assess the efficacy and safety of Danhong injection (DHI) for acute myocardial infarction (AMI) patients. Methods. We searched several electrical databases and hand searched several Chinese medical journals. Randomized controlled trials (RCTs) comparing DHI plus conventional western medicine with conventional western medicine plus placebo and RCTs comparing DHI plus conventional western medicine with conventional western medicine were retrieved. Study screening, data extraction, quality assessment, and data analysis were conducted in accordance with the Cochrane standards. Results. 13 RCTs enrolling 979 patients were included. Danhong injection could significantly reduce the risk of mortality, recurrent angina, arrhythmia, and heart failure. In addition, DHI was associated with improvement of left ventricular ejection fraction (LVEF) and reperfusion. No significant difference of DHI was found on recurrent acute myocardial infarction. However, the safety of DHI remained unknown for limited data. Conclusion. DHI might be a potentially efficacious treatment for AMI patients. Nevertheless, the safety of DHI remained uncertain for limited information. Due to the fact that the overall quality of all included studies is generally low, more high quality RCTs are expected to validate the efficacy and safety of DHI for AMI patients.

\section{Introduction}

Acute myocardial infarction (AMI), a lethal type of coronary heart disease (CHD), is one of the major causes of death in the world [1]. AMI occurs when coronary artery is occluded, usually on the basis of rupture, thrombosis, or erosion of the coronary atherosclerotic plaque, leading to acute inadequate blood flow and oxygen supply to heart muscle [2]. AMI is the most common cause of morbidity among ischemic heart diseases and is the leading death cause in the western world [3]. Even though the application of revascularization, including thrombolytic, percutaneous coronary intervention (PCI) and coronary artery bypass grafting (CABG), has successfully reduced AMI patients' mortality, they are still facing certain risk of in-hospital death [4]. Being in a dilemma, revascularization is also associated with intractable complications, for example, no-reflow phenomenon after
PCI, intrastent thrombosis, and ischemia-reperfusion injury [5]. Since the frequent and successful use of Traditional Chinese Medicine (TCM) in the prevention and treatment for CHD, the effects of TCM for CHD have aroused increasing attention [6-8].

Danhong injection (DHI), a Chinese patent compound injection, is widely used in the treatment for several diseases, including AMI. DHI consists of two components, roots of danshen (Radix Salvia Miltiorrhizae) and flower of honghua (Flos Carthami Tinctorii) [9]. Previous studies have shown that danshen, the main constituent of DHI, could be a vasodilator, lowering the vascular resistance and blood viscosity so as to protect myocardium [10-12]. Moreover, honghua has been demonstrated to possess multiple pharmacological characteristics, including vasodilation, antioxidation, calcium antagonism, and oxygen-free-radical scavenging [13-15]. Recent pharmacological researches have 
also shown that DHI has a positive effect in inhibiting the aggregation of platelet, improving AMI patient's hemodynamic status and endothelial function [16-18]. Meanwhile, a large number of clinical trials also revealed the positive efficacy of DHI for AMI patients. Thus, DHI might be a potentially effective medicine for AMI. However, the efficacy and safety of DHI for AMI have not yet been systematically assessed. So our study aimed to assess the efficacy and safety of DHI plus the conventional treatment for AMI patients.

\section{Methods}

2.1. Inclusion and Exclusion Criteria. All randomized controlled trials (RCTs) comparing DHI plus conventional western medicine with conventional western medicine plus placebo and RCTs comparing Danhong injection plus conventional western medicine with conventional western medicine were included, regardless of their publication status, population characteristics, or languages. Patients meeting any one of the current or previous AMI diagnostic criteria [19-23] were included, with no limitation to their gender, age, religion, or ethnic origin. Trials that did not provide description of diagnostic criteria but declared patients with definite AMI were also considered. Primary outcomes included mortality, recurrent AMI, and reperfusion rate. Secondary outcomes included heart failure, arrhythmia, left ventricular ejection fraction (LVEF), recurrent angina, and adverse events. Quasirandomized controlled trials, animal trials, pharmacological trials, and duplicated trials were excluded. In addition, trials whose difference in sample size between DHI group and control group was greater than $50 \%$ were also excluded so as to ensure the precision of the study.

2.2. Database and Search Strategies. We extensively searched published and unpublished RCTs in the following databases: PubMed, The Cochrane Library (Issue 12, 2014), Embase, Chinese Biomedical Database (CBM), Chinese VIP Information (VIP), China National Knowledge Infrastructure (CNKI), and Wanfang Databases, with search terms adjusted to each database as followed: "Danhong injection," "acute myocardial infarction," "coronary artery disease," "acute coronary syndrome." We searched ongoing registered clinical trials on the website of WHO International Clinical Trial Registry Platform (http://apps.who.int/trialsearch/) and international clinical trial registry by US national institutes of health (http://www.clinicaltrials.gov/). Our entire search was ended on December 28, 2014. Besides, bibliographies of the included studies were searched to avoid missing relevant articles.

2.3. Study Selection and Data Extraction. Two authors (Juming Huang and Ruixiang Zeng) independently scanned the search results by titles and abstracts and selected potentially relevant RCTs. Full texts of potentially relevant articles were retrieved. Based on the inclusion and exclusion criteria, articles were further identified. Data of included studies was extracted and filled into a prespecified electronic form by two authors, Juming Huang and Ruixiang Zeng independently. The extracted data included authors, title, and year of publication, sample size, age and sex of the participants, information of methodological quality, details of the treatment for both groups, outcomes, and adverse effects for each study. Disagreement (if any) was solved by discussions with a third author (Lei Wang).

2.4. Risk of Bias Assessment. In accordance with the Cochrane Collaboration's risk of bias assessment tool [37], two authors (Juming Huang and Ruixiang Zeng) independently evaluated the methodological quality of all included studies via the following aspects: random sequence generation (selection bias), allocation concealment (selection bias), blinding of participants and personnel (performance bias), blinding of outcome assessment (detection bias), incomplete outcome data (attrition bias), selective reporting (reporting bias), and other biases. For each aspect, a low risk was considered when we judged a "Yes," conversely, a "No" for a high risk, and otherwise for an unclear risk. Efforts were made to obtain missing information from the original authors whenever possible. Discrepancies were resolved by consultation with a third author (Lei Wang).

2.5. Data Analysis. Revman 5.3 software from Cochrane Collaboration was applied for data analyses. Dichotomous data were presented as risk ratio (RR) and continuous outcomes as mean difference (MD), both with $95 \%$ confidence interval (CI). We always performed intention-to-treat (ITT) analysis to analyze data whenever possible. Fixed effects model was applied to analyze data if there was low heterogeneity $\left(I^{2} \leq\right.$ $50 \%)$; random effects model was used if there was high heterogeneity $\left(50 \%<I^{2}<75 \%\right)$. Data were not pooled if there was significant heterogeneity $\left(I^{2} \geq 75 \%\right)$ [37], in which case we explored potential causes of heterogeneity by conducting subgroup analyses based on the characteristics of intervention (dosage, duration), the types of conventional therapy (PCI versus thrombolysis), and the methodological quality. Sensitivity analysis was applied on low methodological quality studies in order to investigate whether including such studies would alter the results. Publication biases were explored by funnel plot analysis if the number of included studies of any outcomes was greater than ten [37].

\section{Results}

3.1. Search Flow. A flow diagram demonstrated the search process and study selection (Figure 1). According to the preset search strategy, a total of 543 studies were found, of which 278 were excluded for duplicates. After reading the titles and abstracts, we excluded 125 articles for different reasons. 140 potentially eligible articles were retrieved for further assessment, of which 127 were excluded for the following reasons: being irrelevant to myocardial infarction $(n=90)$, being irrelevant to primary or secondary outcomes $(n=32)$, non-RCTs $(n=3)$, and control group that contained other therapies of Chinese medicine $(n=2)$. Therefore, 13 studies [24-36] were included. We also found one ongoing trail via US national institutes of health. This trial was not included in our review because it was recruiting participants. 


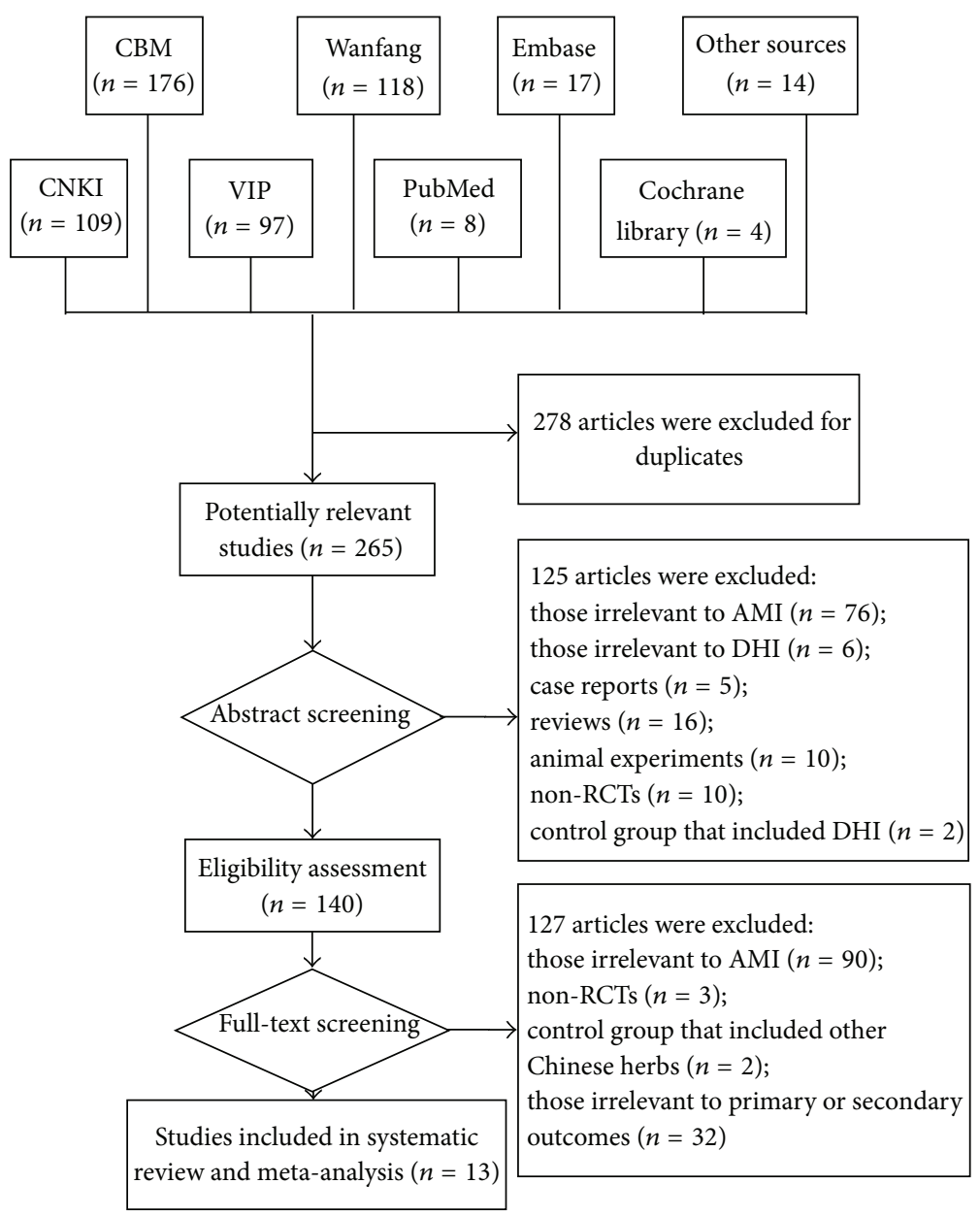

FIGURE 1: Flowchart of study search and identification. Notes: CNKI: China National Knowledge Infrastructure; CBM: Chinese Biomedical Database; VIP: Chinese VIP Information; AMI: acute myocardial infarction; DHI: Danhong injection.

3.2. Description of Included Studies. The characteristics of the 13 included studies [24-36] are summarized in Table 1. All these studies were conducted in China and published in Chinese. Among 13 included studies, two were postgraduate dissertation [33, 34], one was conference proceedings [36], and others were journal articles published from 2008 to 2013. The sample size in each of the included studies ranged from 40 to 134 , with a total of 979 AMI patients in 13 included studies. Male (601) participants were more than female (378) participants. The age of participants was widely distributed, ranging from 36 to 85 years old.

Participants were diagnosed with AMI via different criteria: the CSCCMA diagnostic criteria were used in five studies [30, 32-34, 36]; the WHO diagnostic criteria were used in one study [29]; ACC/AHA diagnostic criteria were used in one study [24] and six studies [25-28, 31, 35] that failed to give a detailed description of their diagnostic criteria but mentioned "participants with AMI were included." STEMI participants were included in two studies [24, 29], NSTEMI participants were included in one study [34], and participants that were included in the other studies were unclear about the types of AMI [25-28, 30-34, 36]. Detailed baseline information was available in six studies [26, 27, 29, 30, 32, 35]. In each of the included studies, baseline difference between experiment group and control group revealed no statistical significance.

All participants in the intervention groups received DHI plus conventional therapy while control groups received conventional treatment. Twelve studies [24-34, 36] specified the doses of DHI they used, ranging from $20 \mathrm{~mL}$ to $40 \mathrm{~mL}$, while one study did not introduce the specific doses [35]. Eleven studies [24-29, 31-34, 36] reported the duration of treatment (from 7 days to 4 weeks) and length of follow-up (from 1 week to 3 months), while the other two studies did not.

Mortality was reported in six studies [25, 27, 30-32, 35]. Recurrent AMI was reported in two studies [25, 31]. Seven studies provided information of reperfusion [26, 27, 30$32,35,36]$. Six studies provided the number of patients with recurrent angina $[24,25,29,32,33,36]$. Arrhythmia was reported in eleven studies [25, 26, 28-36]. Eight studies offered information of participants who suffered from heart failure $[25,26,29-33,35]$. Five studies reported the outcomes of LVEF [26, 28, 31, 34, 36]. As for adverse events, five studies 


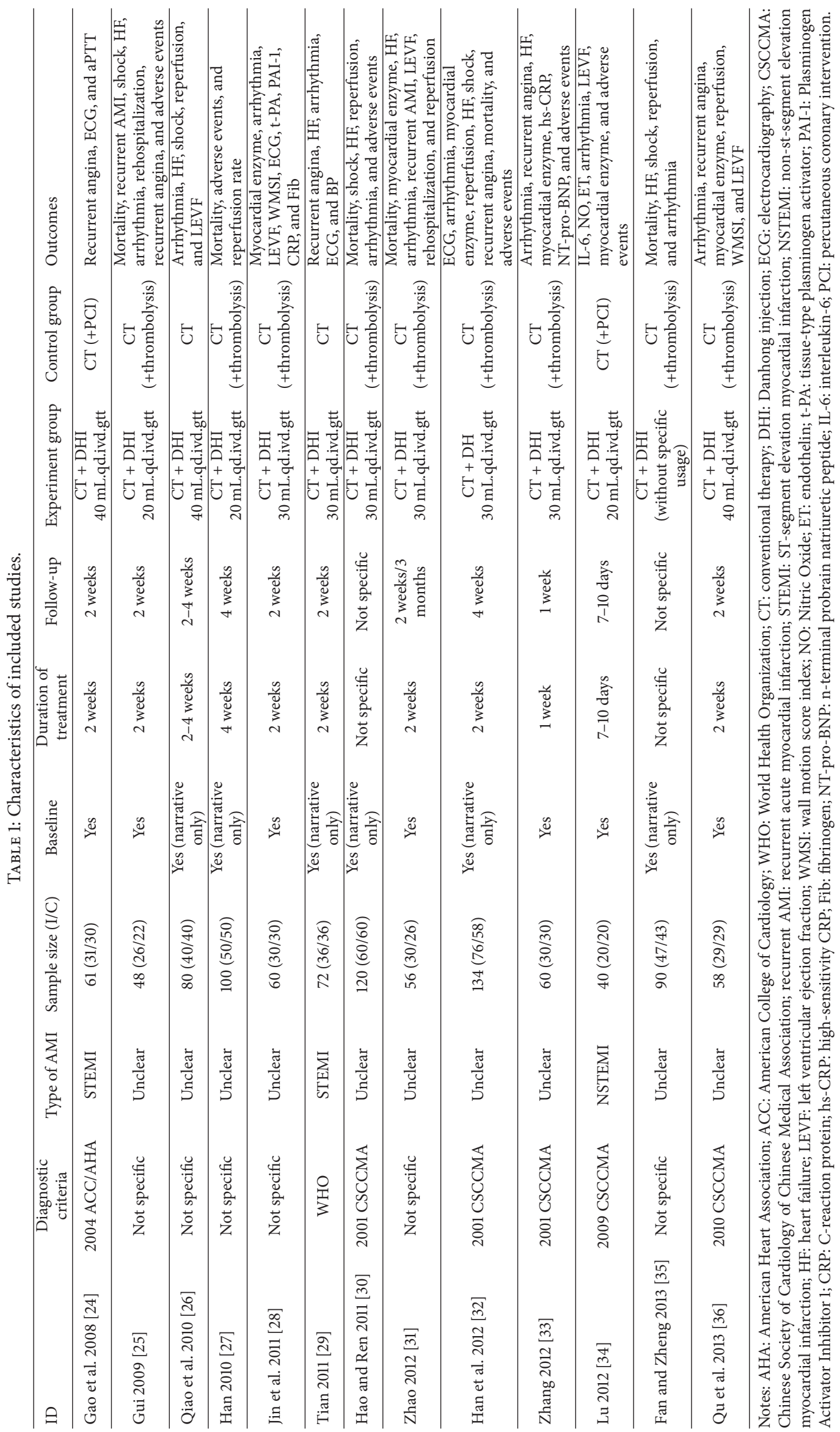




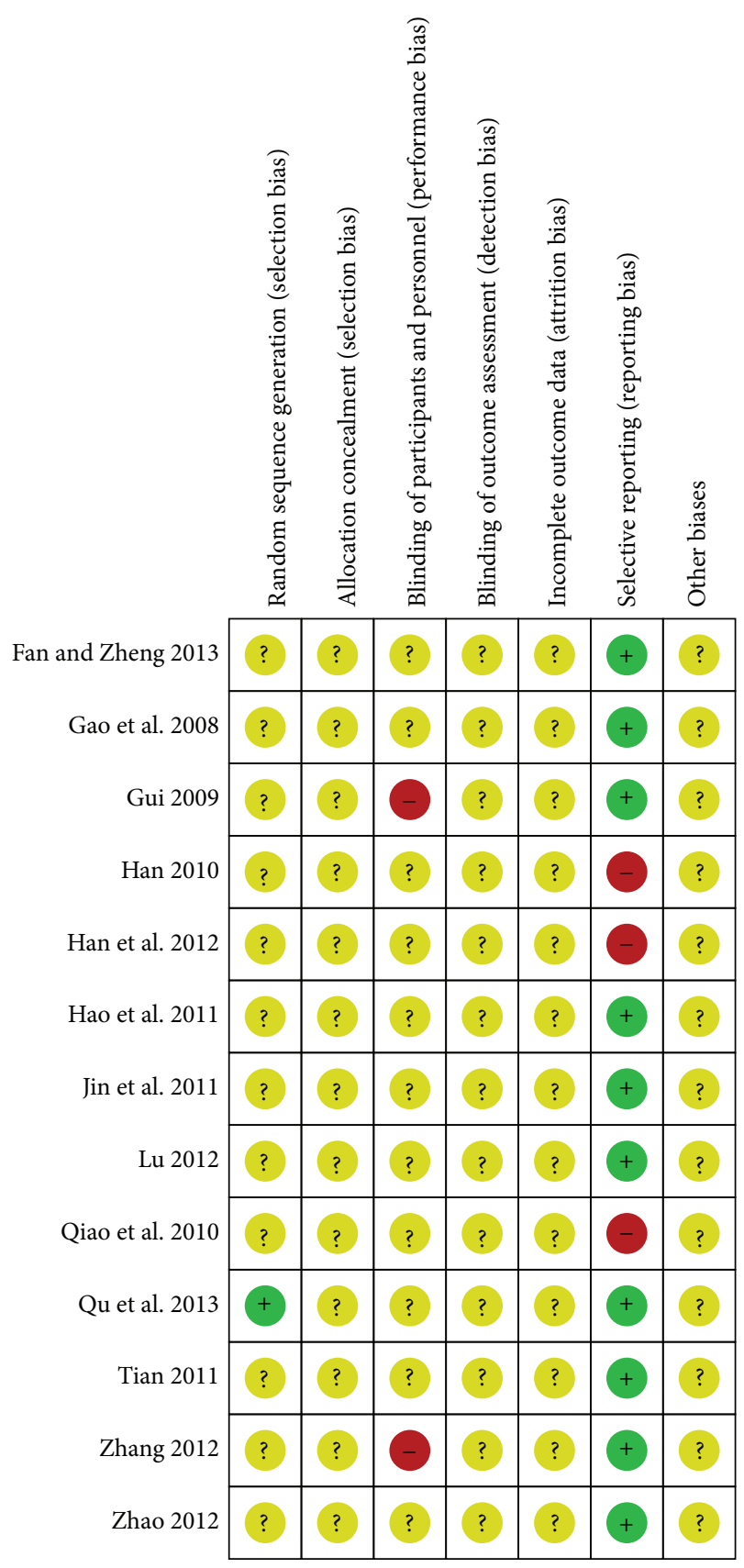

FIGURE 2: Risk of bias summary: review authors' judgment on each risk of bias item for each included study.

$[25,27,30,32,33]$ provided numerical cases on bleeding events and one study [34] gave a narrative introduction of liver and kidney functions.

3.3. Methodological Quality of Included Trials. Risk of bias assessment of all included studies is presented in Figure 2. According to Cochrane Collaboration criteria, all of the thirteen included trials were evaluated as low methodological quality. Only one [36] of the thirteen included studies reported that random sequence was generated from a random number table, while other studies failed to account for the random sequence generation. None of the included studies described the allocation concealment. Two trials [25, 33] mentioned that they were single-blinded and the remaining trials did not report blinding of participants or personnel. Blinding of outcome assessment was not mentioned in any studies. Neither withdrawals nor losses to follow-up were reported in the included studies. Three trials $[26,27,32]$ were considered to be associated with selective outcome reporting, because some outcomes were omitted or incomplete. No study mentioned prior sample size estimation or ITT analysis for any outcome. We tried every effort to contact authors by telephone, email, and other ways for further information about the trials. No other information was obtained. 


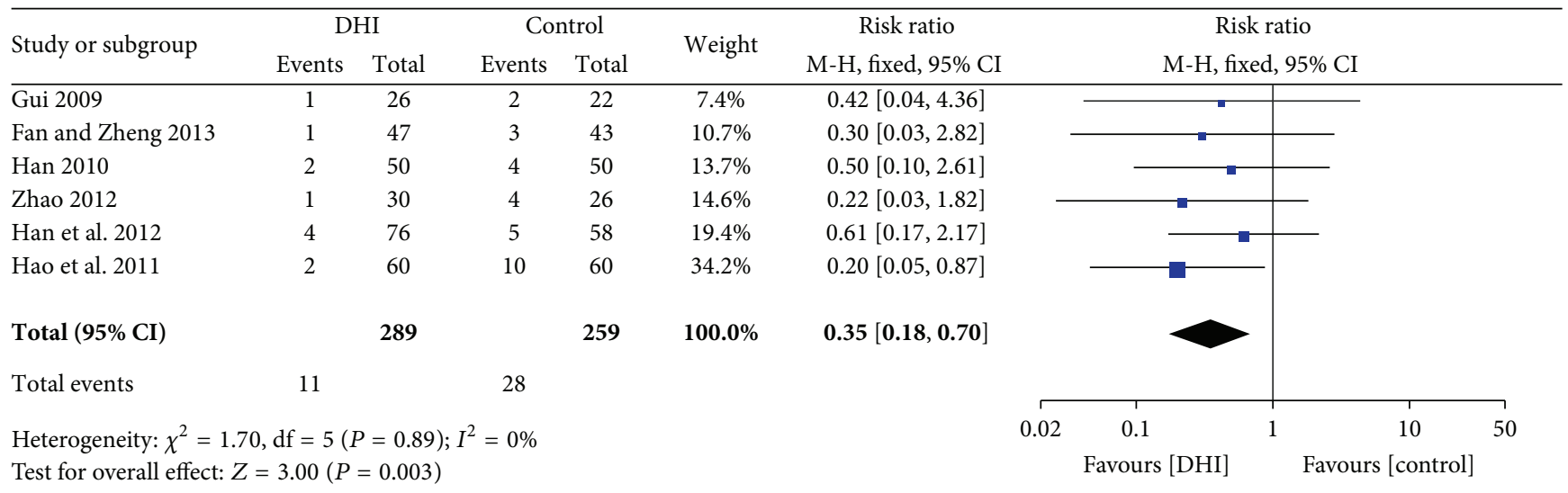

FIgURE 3: Forrest plot of mortality.

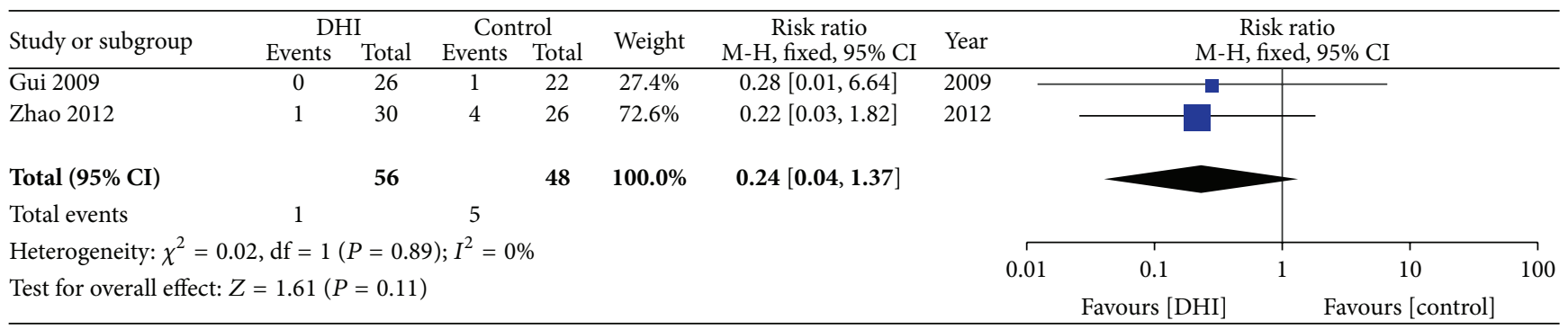

FIGURE 4: Forrest plot of recurrent acute myocardial infarction (AMI).

\subsection{Effect of the Interventions}

3.4.1. Mortality. Six studies [25, 27, 30-32, 35] reported mortality. Meta-analysis showed statistically significant difference in the risk of mortality between conventional treatment plus DHI and conventional treatment (RR: 0.35 ; 95\% CI 0.18 to $0.70 ; n=548 ; I^{2}=0 \%$ ) (Figure 3 ).

3.4.2. Recurrent AMI. Two studies [25, 31] reported recurrent AMI. Meta-analysis showed no statistically significant difference in the risk of recurrent AMI between conventional treatment plus DHI and conventional treatment (RR: 0.24; $95 \%$ CI 0.04 to $1.37 ; n=104 ; I^{2}=0 \%$ ) (Figure 4 ).

3.4.3. Reperfusion. Reperfusion was reported in seven studies [26, 27, 30-32, 35, 36]. Meta-analysis (random effect model) revealed that conventional treatment plus DHI was associated with a statistically significant increase in reperfusion compared with conventional treatment (RR: 1.41; 95\% CI 1.15 to $\left.1.72 ; n=638 ; I^{2}=60 \%\right)$. Since significant heterogeneity was observed, we rechecked these studies carefully and found out the difference of methodological quality among seven studies. Three trials $[26,27,32]$ were assessed as low quality in selective outcome reporting, while the rest of the trials were of high quality in this aspect. Therefore, a subgroup analysis was conducted according to the methodological quality among seven studies. In the low quality subgroup $[26,27,32]$, meta-analysis result (fixed effect model) changed to be statistically insignificant with no heterogeneity (RR: 1.13; 95\% CI 0.96 to $1.33 ; n=314$; three studies; $\left.I^{2}=0 \%\right)$. In the high quality subgroup $[30,31,35,36]$, meta-analysis result (fixed effect model) was still statistically significant with low heterogeneity (RR: 1.82 ; $95 \%$ CI 1.49 to 2.23 ; $n=324$; four studies; $I^{2}=19 \%$ ) (Figure 5).

3.4.4. Recurrent Angina. Six studies [24, 25, 29, 32, 33, 36] assessed recurrent angina. Mete-analysis showed that compared with conventional treatment, conventional treatment plus DHI was associated with a statistically significant decrease in the risk of recurrent angina (RR: 0.41 ; 95\% CI 0.26 to $0.66 ; n=433 ; I^{2}=0 \%$ ) (Figure 6).

3.4.5. Arrhythmia. Eleven trials [25, 26, 28-36] assessed participants who suffered from arrhythmia. The meta-analysis result manifested that conventional treatment plus DHI was associated with a statistically significant decline in the risk of arrhythmia compared with conventional treatment (RR: 0.61; 95\% CI 0.52 to $0.72 ; n=818 ; I^{2}=0 \%$ ) (Figure 7 ). A funnel plot was applied to investigate the publication bias among these studies. The asymmetry of the funnel plot indicated that potential publication bias among studies might influence the result (Figure 8).

3.4.6. Heart Failure. Eight studies reported heart failure [25, 26, 29-33, 35]. Meta-analysis of these studies revealed that conventional treatment plus DHI was associated with 


\begin{tabular}{|c|c|c|c|c|c|c|c|c|}
\hline \multirow{2}{*}{ Study or subgroup } & \multicolumn{2}{|c|}{ DHI } & \multicolumn{2}{|c|}{ Control } & \multirow{2}{*}{ Weight } & \multirow{2}{*}{$\begin{array}{c}\text { Risk ratio } \\
\text { M-H, fixed, 95\% CI }\end{array}$} & \multirow{2}{*}{$\begin{array}{c}\text { Risk ratio } \\
\text { M-H, fixed, 95\% CI }\end{array}$} & Risk of bias \\
\hline & Events & Total & Events & Total & & & & $\begin{array}{lllllll}A & B & C & D & E & F & G\end{array}$ \\
\hline \multicolumn{9}{|l|}{ 1.3.1 Low quality group } \\
\hline Han 2010 & 38 & 50 & 32 & 50 & $19.9 \%$ & $1.19[0.92,1.54]$ & & ? ? ? ? ? ? \\
\hline Han et al. 2012 & 43 & 76 & 31 & 58 & $21.8 \%$ & $1.06[0.78,1.44]$ & & ? ? ? ? ? \\
\hline Qiao et al. 2010 & 31 & 40 & 27 & 40 & $16.8 \%$ & $1.15[0.87,1.51]$ & & ? ? ? ? ? \\
\hline Subtotal (95\% CI) & & 166 & & 148 & $58.5 \%$ & $1.13[0.96,1.33]$ & & \\
\hline Total events & 112 & & 90 & & & & & \\
\hline \multicolumn{9}{|c|}{ Heterogeneity: $\chi^{2}=0.33, \mathrm{df}=2(P=0.85) ; I^{2}=0 \%$} \\
\hline \multicolumn{9}{|c|}{ Test for overall effect: $Z=1.44(P=0.15)$} \\
\hline \multicolumn{9}{|l|}{ 1.3.2 High quality group } \\
\hline Fan and Zheng 2013 & 35 & 47 & 15 & 43 & $9.7 \%$ & $2.13[1.37,3.32]$ & & ? ? ? ? + ? \\
\hline Hao et al. 2011 & 44 & 60 & 21 & 60 & $13.0 \%$ & $2.10[1.44,3.05]$ & & ? ? ? ? + ? \\
\hline Qu et al. 2013 & 29 & 29 & 19 & 29 & $12.1 \%$ & $1.51[1.16,1.98]$ & & + ? ? ? ? ? \\
\hline Zhao 2012 & 16 & 30 & 10 & 26 & $6.7 \%$ & $1.39[0.77,2.50]$ & & ? ? ? ? + ? \\
\hline Subtotal $(95 \%$ CI $)$ & & 166 & & 158 & $41.5 \%$ & $1.82[1.49,2.23]$ & & \\
\hline Total events & 124 & & 65 & & & & & \\
\hline \multirow{2}{*}{\multicolumn{9}{|c|}{$\begin{array}{l}\text { Heterogeneity: } \chi^{2}=3.71, \mathrm{df}=3(P=0.29) ; I^{2}=19 \% \\
\text { Test for overall effect: } Z=5.84(P<0.00001)\end{array}$}} \\
\hline & & & & & & & & \\
\hline Total $(95 \%$ CI $)$ & & 332 & & 306 & $100.0 \%$ & $1.42[1.25,1.61]$ & & \\
\hline Total events & 236 & & 155 & & & & & \\
\hline \multicolumn{9}{|c|}{ Heterogeneity: $\chi^{2}=15.12, \mathrm{df}=6(P=0.02) ; I^{2}=60 \%$} \\
\hline \multicolumn{7}{|c|}{ Test for overall effect: $Z=5.33(P<0.00001)$} & $\begin{array}{lllll}0.5 & 0.7 & 1 & 1.5 & 2\end{array}$ & \\
\hline \multicolumn{7}{|c|}{ Test for subgroup differences: $\chi^{2}=13.05, \mathrm{df}=1(P=0.0003) ; I^{2}=92.3 \%$} & Favours [control] Favours [DHI] & \\
\hline
\end{tabular}

Risk of bias legend

(A) Random sequence generation (selection bias)

(B) Allocation concealment (selection bias)

(C) Blinding of participants and personnel (performance bias)

(D) Blinding of outcome assessment (detection bias)

(E) Incomplete outcome data (attrition bias)

(F) Selective reporting (reporting bias)

(G) Other biases

FIGURE 5: Forrest plot of reperfusion rate.

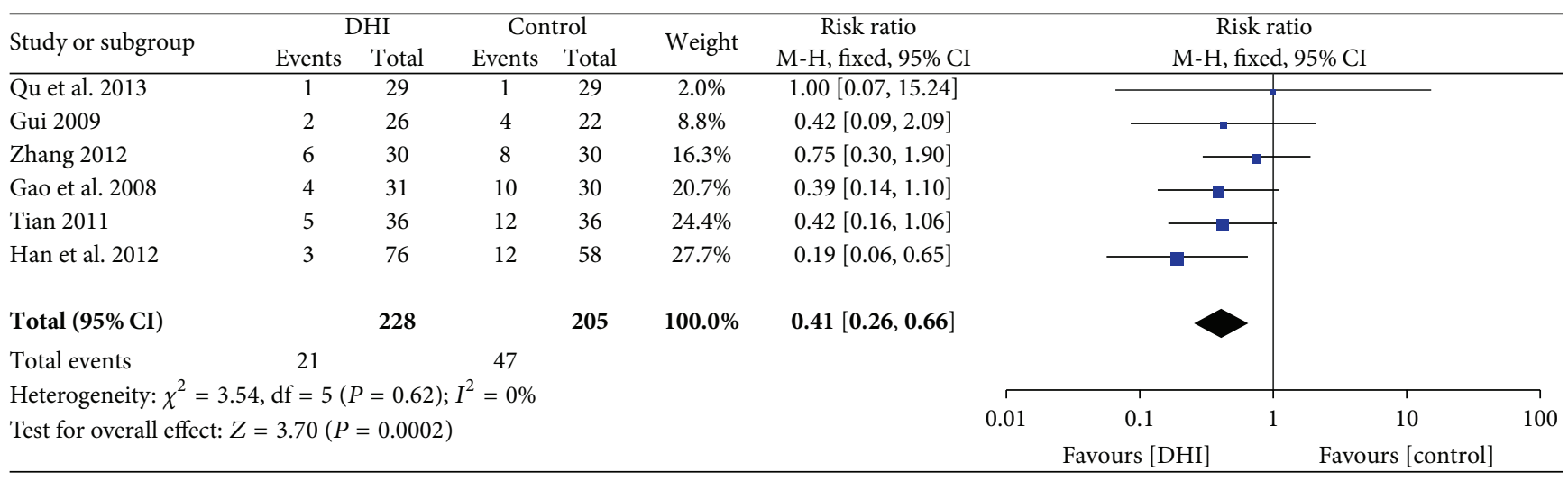

Figure 6: Forrest plot of recurrent angina.

a statistically significant reduction in risk of heart failure (RR: $0.42 ; 95 \%$ CI 0.29 to $0.61 ; n=640 ; I^{2}=0 \%$ ) (Figure 9).

3.4.7. LVEF. Five studies reported the outcomes of LVEF $[26,28,31,34,36]$. Meta-analysis showed that conventional treatment plus DHI was associated with a statistically significant increase in LVEF compared with conventional treatment (MD: $5.21 ; 95 \%$ CI 3.62 to $6.81 ; n=289 ; I^{2}=26 \%$ ) (Figure 10 ).
3.4.8. Adverse Events. Six of the thirteen studies reported adverse events, while thirteen trials did not provide any related information [25, 27, 30, 32-34]. Among six studies, one trial [34] gave a narrative description of liver and kidney functions, showing no statistically significant difference between two groups. Other five studies [25, 27, 30, 32, 33] provided numerical cases of bleeding events in both groups. Therefore, a meta-analysis was performed on bleeding events, 


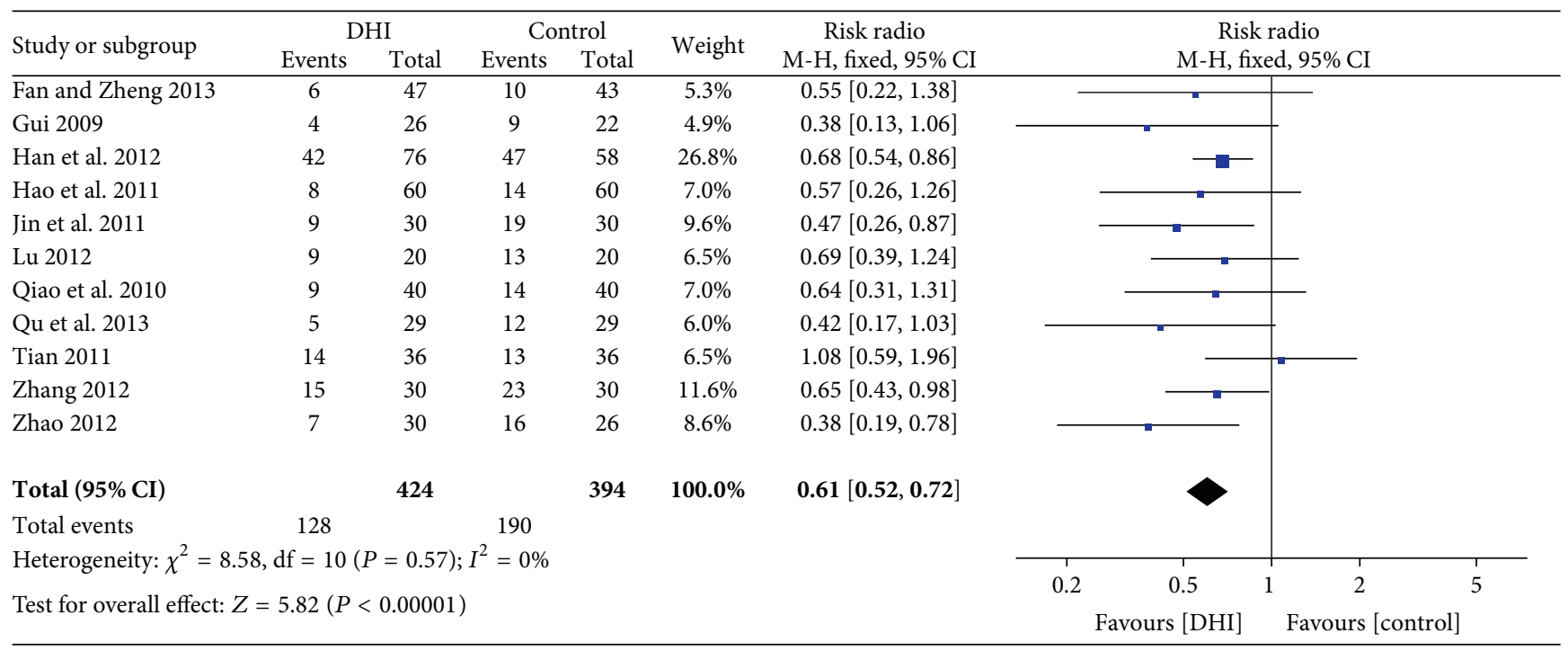

FIGURE 7: Forrest plot of arrhythmia.

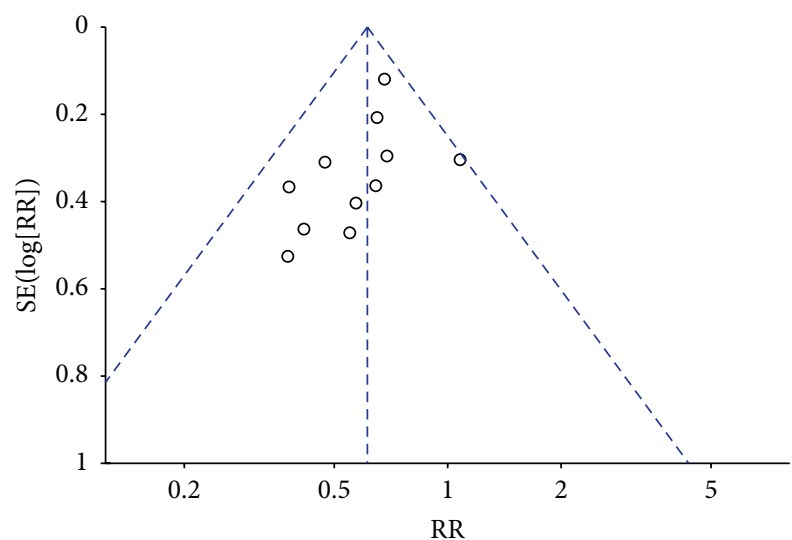

Figure 8: Funnel plot of arrhythmia.

which found that DHI did not increase the risk of bleeding (RR: $1.2595 \%$ CI 0.66 to $2.35 ; n=462 ; I^{2}=0 \%$ ) (Figure 11).

\section{Discussion}

Western medicine, whose aim is to restore blood flow to the ischemic myocardium, has made significant progress in the treatment of AMI with revascularization in past few decades and has tremendously reduced the death risk in AMI patients. However, the recent PEACE study found that the application of western medicine and modern technology such as PCI did not improve the mortality in China in the past decade [38]. Moreover, current studies have found that reperfusion to the ischemic myocardium can paradoxically reduce the beneficial effects of restored blood flow and even aggravate the necrosis of ischemic myocardium, leading to severe complications [39]. Chinese herbal medicinals have been widely applied in the treatment for disorders related to AMI in China since thousands of years ago and were regarded as natural products with better efficacy and less side effects. In china, DHI was one of the most widely used traditional Chinese herbal medicinals for AMI. Several researches implicated that DHI could inhibit the platelet activation and aggregation [40-42], which both play an important role in the process of acute myocardial infarction [43-45]. Recent studies have also found that DHI could protect ischemic myocardium against myocardial ischemia/reperfusion injury [46-48]. Plenty of clinical studies have reported the efficacy of DHI for AMI patients. Based on the previous evidence, a hypothesis was proposed that DHI might be a potentially effective drug in treating AMI patients. However, the efficacy and safety of DHI in treating AMI patients have not yet been critically evaluated. So systematical assessment of the efficacy and safety of DHI for AMI is significantly urgent and necessary.

In this systematic review, thirteen studies were included with a total of 979 participants. There was no statistically significant effect of conventional treatment plus DHI on recurrent AMI. However, conventional treatment plus DHI demonstrated statistically significant benefit in terms of mortality, reperfusion rate, arrhythmia, recurrent angina, heart failure, and improved LVEF as compared with conventional treatment.

In this review, DHI was found with no effect to increase the bleeding risk. However, due to the low quality of the included trials and inadequate data, we are unable to evaluate the safety of DHI for AMI patients at present. Hence, we appeal for a detailed description of adverse events in the future studies of DHI.

A number of limitations should be taken into consideration when accepting the findings of this review. Firstly, none of the thirteen included studies was assessed to be at low risk of bias. Thirteen trials claimed to have performed randomization, but only one [36] trial reported how their random sequence was generated and the rest did not. No study gave any information about allocation concealment. Thus, whether the randomization was effectively conducted 


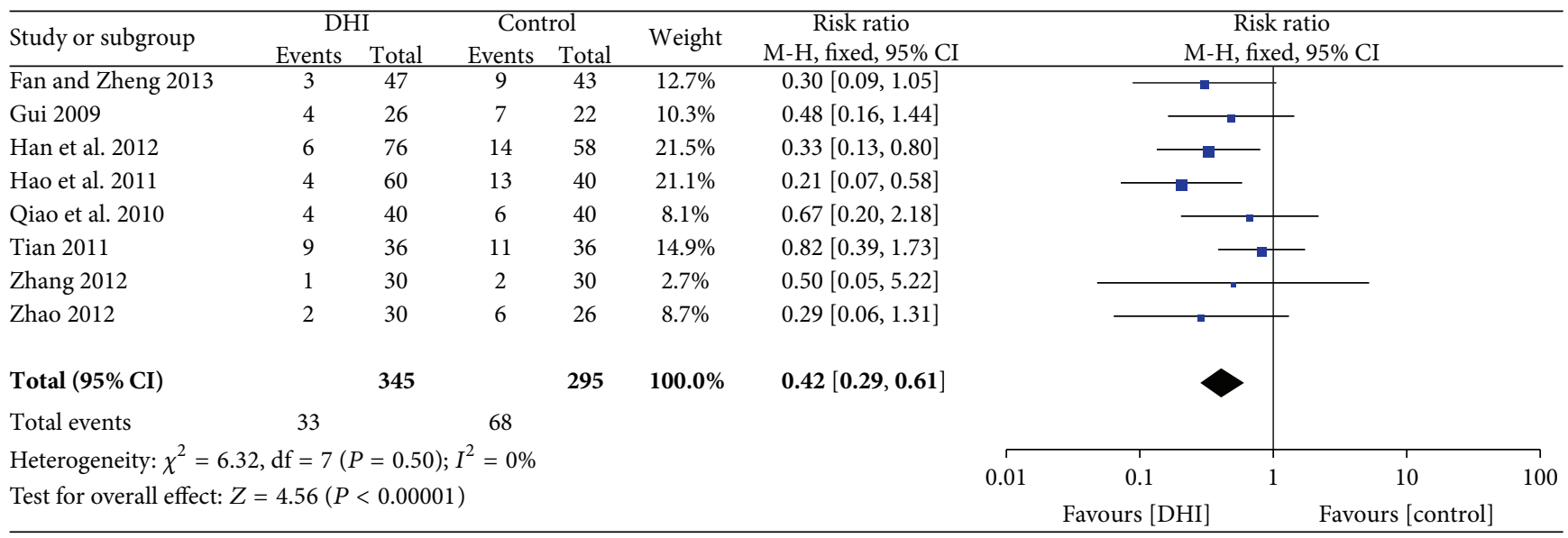

FIGURE 9: Forrest plot of heart failure.

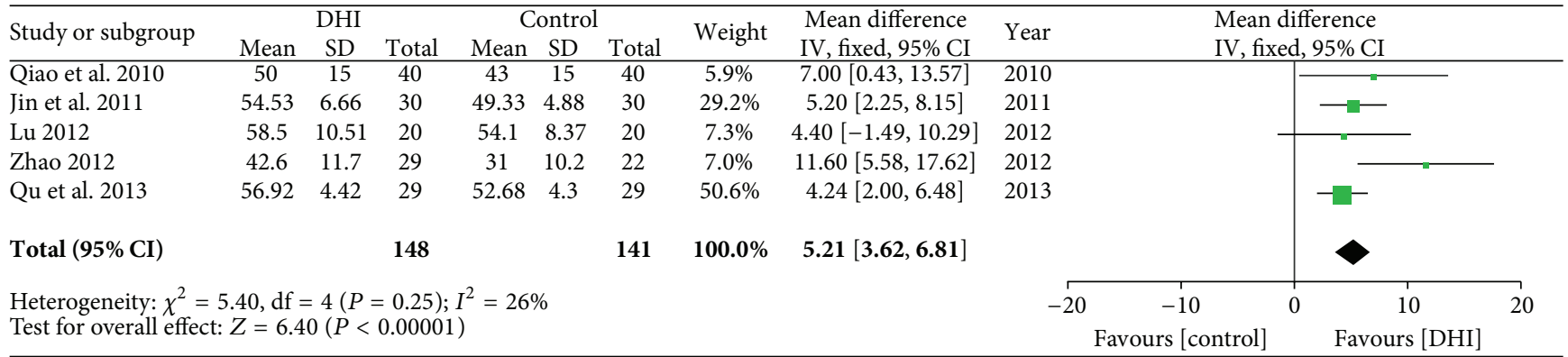

FIGURE 10: Forrest plot of left ventricular ejection fraction (LVEF).

in these trials was doubtful, which might lead to potential selection bias. Two studies [25, 33] were single-blinded while the others did not report blinding of participants or personnel. In all studies, the blinding of outcome assessment remained unknown. Insufficient reporting of blinding on participants, personnel, and outcome assessors might lead to potential performance bias and detection bias. Dropout account, withdrawals, and ITT analysis were not reported in any study, which might lead to potential attrition bias. Three studies $[26,27,32]$ had high risk at selective reporting, which might lead to potential reporting bias. In addition, none of the included studies has reported the sample size estimation and most of the durations of follow-up were short, which weakened the validity of statistical analysis. Since the low quality of the included studies, the widespread popularity of traditional Chinese medicines, and the preference on traditional Chinese medicines of Chinese patients, it is hard to distinguish whether the effect of DHI was confounded by other traditional Chinese medicines. Therefore, we cannot draw a convincing conclusion that there were significant beneficial effects of DHI combined with conventional treatment compared with conventional treatment alone. Secondly, although extensive research was performed in several databases without setting any language limitation, all of the included studies in this review were published in Chinese, which lead to potential location bias. Thus, the asymmetry of the funnel plot indicated that the publication bias could not be excluded. Hence, reporting bias might exist in this review and exaggerate the results. Thirdly, except one study [33], the rest included studies failed to provide a detailed descriptions of their conventional treatment. Differences of conventional treatment among included studies contain potential confounding factors, which might influence the results of meta-analysis and reduce the validity of this review (e.g., angiotensin converting enzyme inhibitor was used in one trial as conventional treatment, while others did not. Meta-analysis might show a statistical difference. Statistical difference may not be caused by intervention but by confounding factor (ACEIs)). Fourthly, previous studies have demonstrated that physical activity and exercise appear to be beneficial for AMI patients [49-51]. Tai Chi, or Tai Chi Chuan, which was an exercise that originated from ancient Chinese martial arts and traditional Chinese medical theory, was also validated to have positive effect on patients with different diseases [52-54], including AMI patients $[55,56]$. Since Tai Chi was widely popular among Chinese patients and it was easy to perform. However, in high quality and rigorous RCTs, it was intractable to detect it or to control it, not to mention in our poor quality included studies. Therefore, exercise like Tai Chi might be a potential confounder that might have an impact on our study results. 


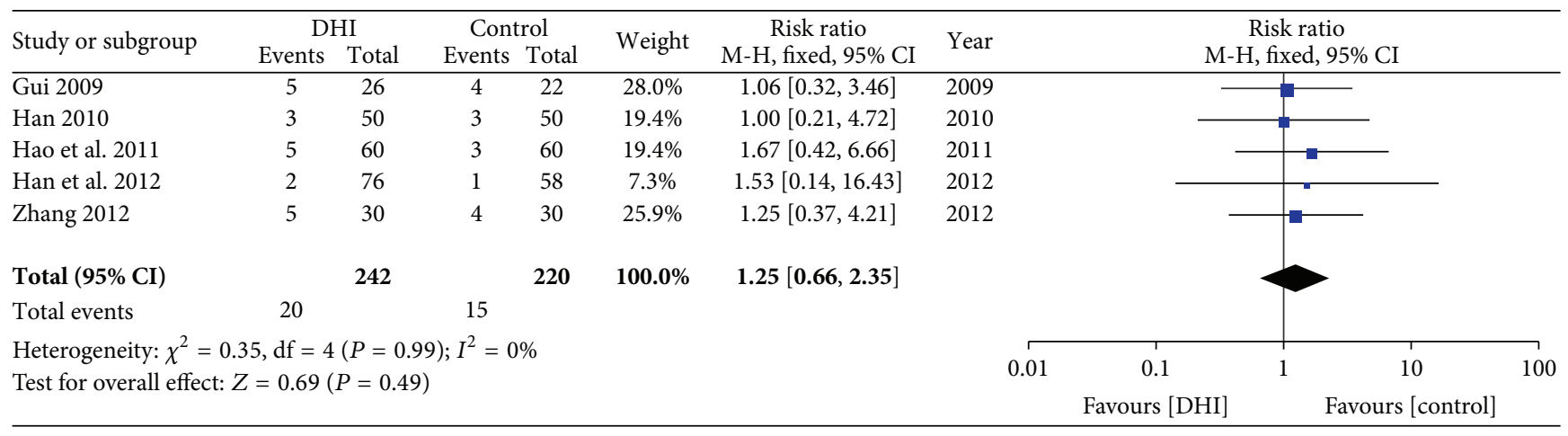

FIGURE 11: Forrest plot of bleeding events.

\section{Conclusion}

This systematic review finds potential benefits of DHI on AMI patients in terms of the incidence of mortality, reperfusion rate, arrhythmia, recurrent angina, heart failure, and LVEF, as compared with conventional treatment. However, the benefits should be cautiously considered due to the poor quality of evidence. In addition, the safety of DHI has not yet been verified for the deficiency of available studies. More high quality evidence from high quality RCTs is needed to support the clinical use of DHI for AMI patients.

\section{Conflict of Interests}

The authors declare that there is no conflict of interests regarding the publication of this paper.

\section{Authors' Contribution}

Pengda Liao, Lei Wang, and Minzhou Zhang conceived and designed the experiment. Pengda Liao and Lei Wang developed the search strategy and data extraction form and drafted the paper. Articles searching, search result screening, data extraction, and risk of bias assessment were performed by Juming Huang and Ruixiang Zeng. Data verification and analysis were carried out by Pengda Liao, Lei Wang, and Liheng Guo. Minzhou Zhang provided methodological perspectives and revised the paper. Pengda Liao and Lei Wang contributed equally to this study.

\section{Acknowledgments}

This research was funded by the Department of Science and Technology and the Academy of Traditional Chinese Medicine of Guangdong Province (Program no. 2014A020221037 and no. 2011B032200006).

\section{References}

[1] H. D. White and D. P. Chew, "Acute myocardial infarction," The Lancet, vol. 372, no. 9638, pp. 570-584, 2008.

[2] P. Libby, "Current concepts of the pathogenesis of the acute coronary syndromes," Circulation, vol. 104, no. 3, pp. 365-372, 2001.
[3] World Bank, Toward a Healthy and Harmonious Life in China: Stemming the Rising Tide of Non-Communicable Diseases, World Bank, Washington, DC, USA, 2011.

[4] X.-J. Ma, H.-J. Yin, and K.-J. Chen, "Appraisal of the prognosis in patients with acute myocardial infarction treated with primary percutaneous coronary intervention," Chinese Journal of Integrative Medicine, vol. 15, no. 3, pp. 236-240, 2009.

[5] A. Tanaka, T. Kawarabayashi, Y. Nishibori et al., "No-reflow phenomenon and lesion morphology in patients with acute myocardial infarction," Circulation, vol. 105, no. 18, pp. 2148 2152, 2002.

[6] H.-X. Liu, S.-R. Wang, Y. Lei, and J.-J. Shang, "Characteristics and advantages of traditional Chinese medicine in the treatment of acute myocardial infarction," Journal of Traditional Chinese Medicine, vol. 31, no. 4, pp. 269-272, 2011.

[7] K.-J. Chen, K. K. Hui, M. S. Lee, and H. Xu, "The potential benefit of complementary/alternative medicine in cardiovascular diseases," Evidence-Based Complementary and Alternative Medicine, vol. 2012, Article ID 125029, 1 page, 2012.

[8] G. Dobos and I. Tao, "The model of Western integrative medicine: the role of Chinese medicine," Chinese Journal of Integrative Medicine, vol. 17, no. 1, pp. 11-20, 2011.

[9] M. Sun, J.-J. Zhang, J.-Z. Shan et al., "Clinical observation of Danhong Injection (herbal TCM product from Radix Salviae miltiorrhizae and Flos Carthami tinctorii) in the treatment of traumatic intracranial hematoma," Phytomedicine, vol. 16, no. 8, pp. 683-689, 2009.

[10] T. O. Cheng, "Cardiovascular effects of Danshen," International Journal of Cardiology, vol. 121, no. 1, pp. 9-22, 2007.

[11] F. F. Y. Lam, J. H. K. Yeung, K. M. Chan, and P. M. Or, "Relaxant effects of danshen aqueous extract and its constituent danshensu on rat coronary artery are mediated by inhibition of calcium channels," Vascular Pharmacology, vol. 46, no. 4, pp. 271-277, 2007.

[12] F. F. Y. Lam, J. H. K. Yeung, Y. W. Kwan, K. M. Chan, and P. M. Y. Or, "Salvianolic acid B, an aqueous component of danshen (Salvia miltiorrhiza), relaxes rat coronary artery by inhibition of calcium channels," European Journal of Pharmacology, vol. 553, no. 1-3, pp. 240-245, 2006.

[13] X. Su, Y. He, W. Yang, Y. Wang, W. Zhang, and Y. Wang, "Effect of dan hong injection on PON1, SOD activity and mda levels in elderly patients with coronary heart disease," International Journal of Clinical and Experimental Medicine, vol. 7, no. 12, pp. 5886-5889, 2014. 
[14] S.-Y. Han, H.-X. Li, X. Ma, K. Zhang, Z.-Z. Ma, and P.-F. Tu, "Protective effects of purified safflower extract on myocardial ischemia in vivo and in vitro," Phytomedicine, vol. 16, no. 8, pp. 694-702, 2009.

[15] X. Zhou, L. Tang, Y. Xu, G. Zhou, and Z. Wang, "Towards a better understanding of medicinal uses of Carthamus tinctorius L. in traditional Chinese medicine: a phytochemical and pharmacological review," Journal of Ethnopharmacology, vol. 151, no. 1, pp. 27-43, 2014.

[16] Y. Q. Jiang, "Effect of danhong injection of patients with coronary artery disease on platelet activation and blood coagulation function," China Modern Doctor, no. 14, pp. 68-70, 2011.

[17] S. J. Li, Y. P. Tang, J. Shen, J. P. Li, G. J. Ming, and J. A. Duan, "Research of Chinese medicine pairs (VIII)—salviae miltiorrhizae radix et rhizoma-carthami flos," China Journal of Chinese Materia Medica, vol. 24, 2013.

[18] G. F. Guan, X. P. Hua, L. Wang, L. Du, and X. H. Kong, "The effects of Danhong injection on vascular inflammation in rabbit model with atherosclerosis," Chinese Journal of Integrative Medicine on Cardio-/Cerebrovascular Disease, vol. 10, pp. 304306, 2006.

[19] Chinese Society of Cardiology of Chinese Medical Association and Editorial Board of Chinese Journal of Cardiology, "Guideline for diagnosis and treatment of patients with unstable angina and non-ST-segment elevation myocardial infarction," Chinese Journal of Cardiology, vol. 35, no. 4, pp. 295-304, 2007.

[20] Chinese Society of Cardiology of Chinese Medical Association and Editorial Board of Chinese Journal of Cardiology, "Recommendation of the application of universal definition of myocardial infarction in China," Chinese Journal of Cardiology, vol. 36, no. 10, pp. 867-869, 2008.

[21] China Society of Cardiology of Chinese Medical Association and Editorial Board of Chinese Journal of Cardiology, "Guideline for diagnosis and treatment of patients with ST-elevation myocardial infarction," Chinese Journal of Cardiology, vol. 38, no. 8, pp. 675-690, 2010.

[22] H. Jneid, J. L. Anderson, R. S. Wright et al., "2012 ACCF/AHA focused update of the guideline for the management of patients with unstable angina/non-ST-elevation myocardial infarction (updating the 2007 guideline and replacing the 2011 focused update): a report of the American College of Cardiology Foundation/American Heart Association Task Force on Practice Guidelines," Journal of the American College of Cardiology, vol. 60, no. 7, pp. 645-681, 2012.

[23] E. A. Amsterdam, N. K. Wenger, R. G. Brindis et al., "2014 AHA/acc guideline for the management of patients with NonST-Elevation acute coronary syndromes: a report of the American College of Cardiology/American Heart Association Task Force on Practice Guidelines," Journal of the American College of Cardiology, vol. 64, no. 24, pp. e139-e228, 2014.

[24] X. D. Gao, P. S. Zhou, and W. W. Kang, "Clinical effect of emergency interventional therapy combined with Danhong injection in the treatment of acute myocardial infarction," Practical Journal of Cardiac Cerebral Pneumal and Vascular Disease, no. 6, pp. 28-29, 2008.

[25] X. Gui, "Research on Danhong injection combined with thrombolysis for acute myocardial infarction," Journal of Medical Forum, no. 21, pp. 73-74, 2009.

[26] "Effect of Danhong injection combined with urokinase thrombolysis for 80 acute myocardial infarction patients," in Proceedings of the 1st National Critical Care Medicine Academic Conference of Integrated Traditional Chinese and Western Medicine and the Founding Conference of Chinese Critical Care Medicine Professional Committe of Integrated Traditional Chinese and Western Medicine, Z. R. Qiao, Q. J. Dong, S. L. Du, C. L. Yuan, and Y. J. Xue, Eds., Guangzhou, China, 2010.

[27] J. X. Han, "Effect of Danhong injection combined with thrombolytic therapy on reperfusion in early acute myocardial infarction," Chinese Journal of Misdiagnostics, no. 15, p. 3556, 2010.

[28] Y. J. Jin, Z. X. Li, and L. Z. Jin, "Myocardial protection effects of Danhong injection in acute myocardial infarction patients receiving thrombolytic therapy for recanalization," Chinese General Practice, no. 32, pp. 3678-3680, 2011.

[29] H. M. Tian, "Effect of Danhong injection in the treatment of acute ST segment elevated myocardial infarction," Liaoning Journal of Traditional Chinese Medicine, no. 11, pp. 2206-2207, 2011.

[30] X. Y. Hao and L. Ren, "Danhong injection combined with urokinase thrombolysis therapy for 60 early acute myocardial infarction patients," Shanxi Journal of Traditional Chinese Medicine, no. 10, pp. 1284-1285, 2011.

[31] S. R. Zhao, "Investigation of short and long term myocardial protection of Danhong injection on thrombolysis patients with acute myocardial infarction," Hebei Journal of Traditional Chinese Medicine, no. 10, pp. 1528-1530, 2012.

[32] G. J. Han, W. H. Liu, D. W. Yang, Z. Y. Gao, J. W. Cao, and L. Y. Zhang, "Effect observation of Danhong injection acute myocardial infarction and myocardial ischemia/reperfusion injury," People's Military Surgeon, no. 6, pp. 514-515, 2012.

[33] G. S. Zhang, The clinical research on reperfusion trauma after thrombolysis in acute myocardial infarction of Qi deficiency and blood stasis syndrome by the effect of Danhong injection [Ph.D. thesis], Fujian University of Traditional Chinese Medicine, Fujian, China, 2012.

[34] J. J. Lu, The Protective Effects and Mechanism of Danhong Injection on Myocardial Ischemia-Reperfusion Injury, Nanjing University of Chinese Medicine, Nanjing, China, 2012.

[35] H. F. Fan and G. Zheng, "Effect of Danhong injection combined with urokinase thrombolysis for early acute myocardial infarction patients," Modern Diagnosis \& Treatment, no. 18, p. 4123, 2013.

[36] Y. N. Qu, C. Y. Yu, and F. X. Ren, "Observation of Danhong Injection's short term effect on st-segment elevated acute myocardial infarction patients with different GRADE scores," Chinese Journal of Integrative Medicine on Cardio-/Cerebrovascular Disease, no. 11, pp. 1321-1322, 2013.

[37] J. P. T. Higgins and S. Green, Cochrane Handbook for Systematic Reviews of Interventions Version 5.1.0, The Cochrane Collaboration, 2011.

[38] J. Li, X. Li, Q. Wang et al., "ST-segment elevation myocardial infarction in China from 2001 to 2011 (the China PEACERetrospective Acute Myocardial Infarction Study): a retrospective analysis of hospital data," The Lancet, vol. 385, no. 9966, pp. 441-451, 2015.

[39] K. Matsumura, R. W. Jeremy, J. Schaper, and L. C. Becker, "Progression of myocardial necrosis during reperfusion of ischemic myocardium," Circulation, vol. 97, no. 8, pp. 795-804, 1998.

[40] M. Cao, X. Lv, Y. He, and K. Liao, "Effect of Danhong injection in hemorheology in old patients with acute coronary syndrome," Practical Journal of Cardiac Cerebral Pneumal and Vascular Disease, vol. 18, pp. 541-542, 2010.

[41] Z.-Q. Chen, L. Hong, and H. Wang, "Effect of danhong injection on platelet activation and inflammatory factors in patients of 
acute coronary syndrome after intervention therapy," Chinese Journal of Integrated Traditional and Western Medicin, vol. 29, no. 8, pp. 692-694, 2009.

[42] D. Wang, G. Fan, Y. Wang et al., "Vascular reactivity screen of Chinese medicine danhong injection identifies Danshensu as a NO-independent but PGI2-mediated relaxation factor," Journal of Cardiovascular Pharmacology, vol. 62, no. 5, pp. 457-465, 2013.

[43] M. D. Trip, V. Manger Cats, F. J. L. van Capelle, and J. Vreeken, "Platelet hyperreactivity and prognosis in survivors of myocardial infarction," The New England Journal of Medicine, vol. 322, no. 22, pp. 1549-1554, 1990.

[44] J. R. O'Brien, J. B. Heywood, and J. A. Heady, "The quantitation of platelet aggregation induced by four compounds: a study in relation to myocardial infarction," Thrombosis et Diathesis Haemorrhagica, vol. 16, no. 3, pp. 752-767, 1966.

[45] M. I. Furman, M. R. Barnard, L. A. Krueger et al., "Circulating monocyte-platelet aggregates are an early marker of acute myocardial infarction," Journal of the American College of Cardiology, vol. 38, no. 4, pp. 1002-1006, 2001.

[46] Y. Guan, Y. Yin, Y.-R. Zhu et al., "Dissection of mechanisms of a Chinese medicinal formula: danhong injection therapy for myocardial ischemia/reperfusion injury in vivo and in vitro," Evidence-Based Complementary and Alternative Medicine, vol. 2013, Article ID 972370, 12 pages, 2013.

[47] J. Liu, D. Zhang, J. Li et al., "Effects of Salvia miltiorrhiza and Carthamus tinctorius aqueous extracts and compatibility on rat myocardial ischemic reperfusion injury," Zhongguo Zhongyao Zazhi, vol. 36, no. 2, pp. 189-194, 2011.

[48] X.-J. Ma, S.-J. Yin, J.-C. Jin et al., "Synergistic protection of danhong injection and ischemic postconditioning on myocardial reperfusion injury in minipigs," Chinese Journal of Integrative Medicine, vol. 16, no. 6, pp. 531-536, 2010.

[49] A. S. Leon, "Exercise following myocardial infarction: current recommendations," Sports Medicine, vol. 29, no. 5, pp. 301-311, 2000.

[50] G. T. O’Connor, J. E. Buring, S. Yusuf et al., "An overview of randomized trials of rehabilitation with exercise after myocardial infarction," Circulation, vol. 80, no. 2, pp. 234-244, 1989.

[51] P. Theroux, D. D. Waters, C. Halphen, J. C. Debaisieux, and H. F. Mizgala, "Prognostic value of exercise testing soon after myocardial infarction," The New England Journal of Medicine, vol. 301, no. 7, pp. 341-345, 1979.

[52] G. Y. Yeh, C. Wang, P. M. Wayne, and R. S. Phillips, "The effect of Tai Chi exercise on blood pressure: a systematic review," Preventive Cardiology, vol. 11, no. 2, pp. 82-89, 2008.

[53] M. S. Lee, M. H. Pittler, and E. Ernst, "Tai chi for osteoarthritis: a systematic review," Clinical Rheumatology, vol. 27, no. 2, pp. 211-218, 2008.

[54] C. Wang, R. Bannuru, J. Ramel, B. Kupelnick, T. Scott, and C. H. Schmid, "Tai Chi on psychological well-being: systematic review and meta-analysis," BMC Complementary and Alternative Medicine, vol. 10, article 23, 2010.

[55] K. S. Channer, D. Barrow, R. Barrow, M. Osborne, and G. Ives, "Changes in haemodynamic parameters following Tai Chi Chuan and aerobic exercise in patients recovering from acute myocardial infarction," Postgraduate Medical Journal, vol. 72, no. 848, pp. 349-351, 1996.

[56] R. M. Nery, M. Zanini, J. B. de Lima, R. P. Bühler, A. D. da Silveira, and R. Stein, "Tai Chi Chuan improves functional capacity after myocardial infarction: a randomized clinical trial," American Heart Journal, vol. 169, no. 6, pp. 854-860, 2015. 


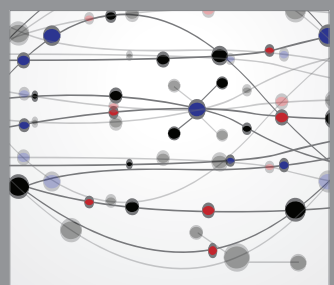

The Scientific World Journal
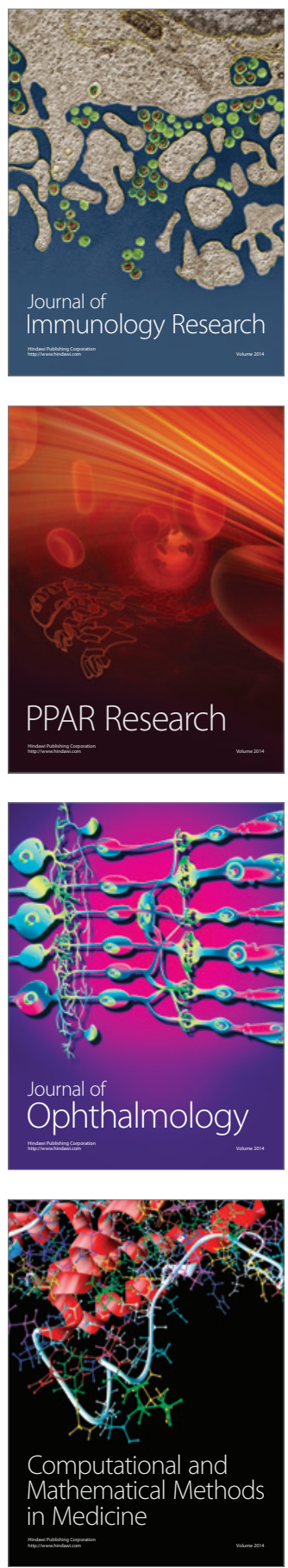

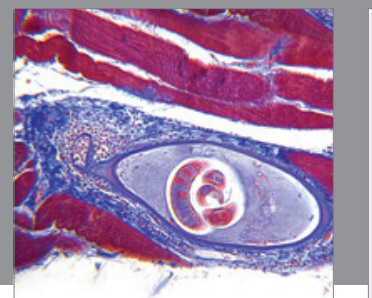

Gastroenterology

Research and Practice
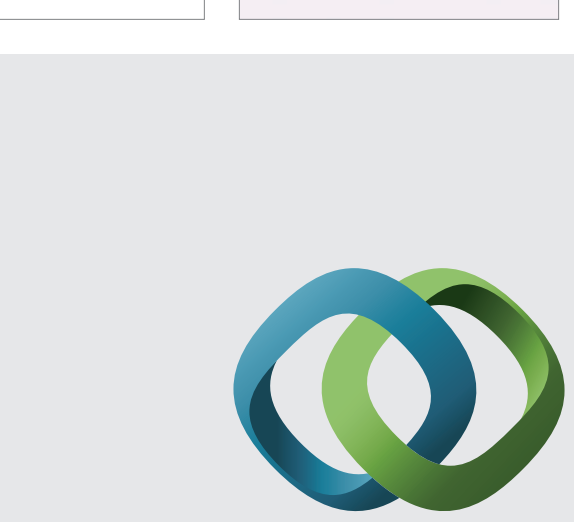

\section{Hindawi}

Submit your manuscripts at

http://www.hindawi.com
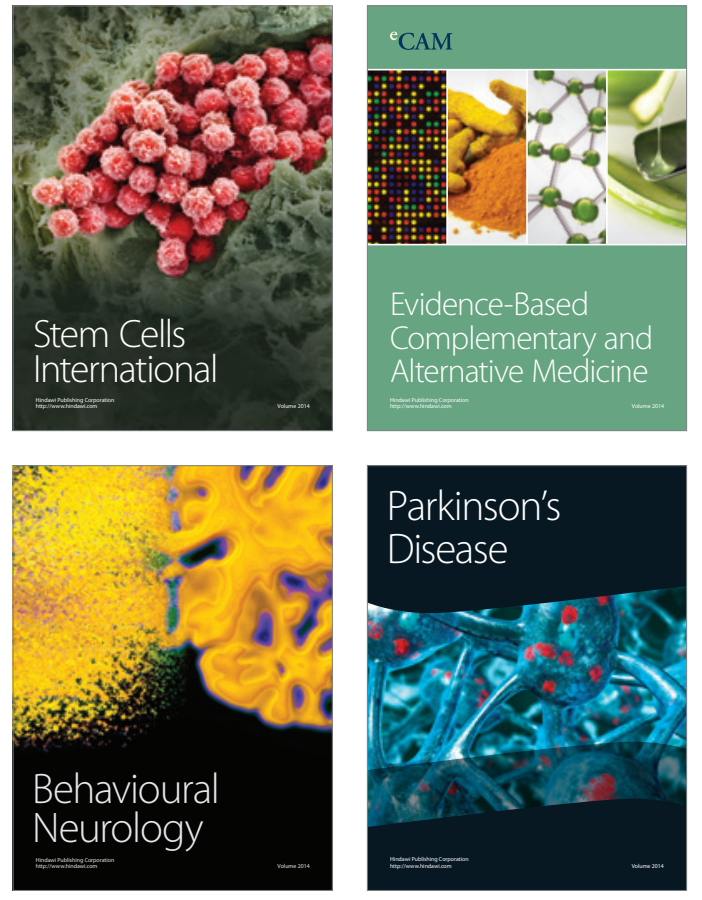
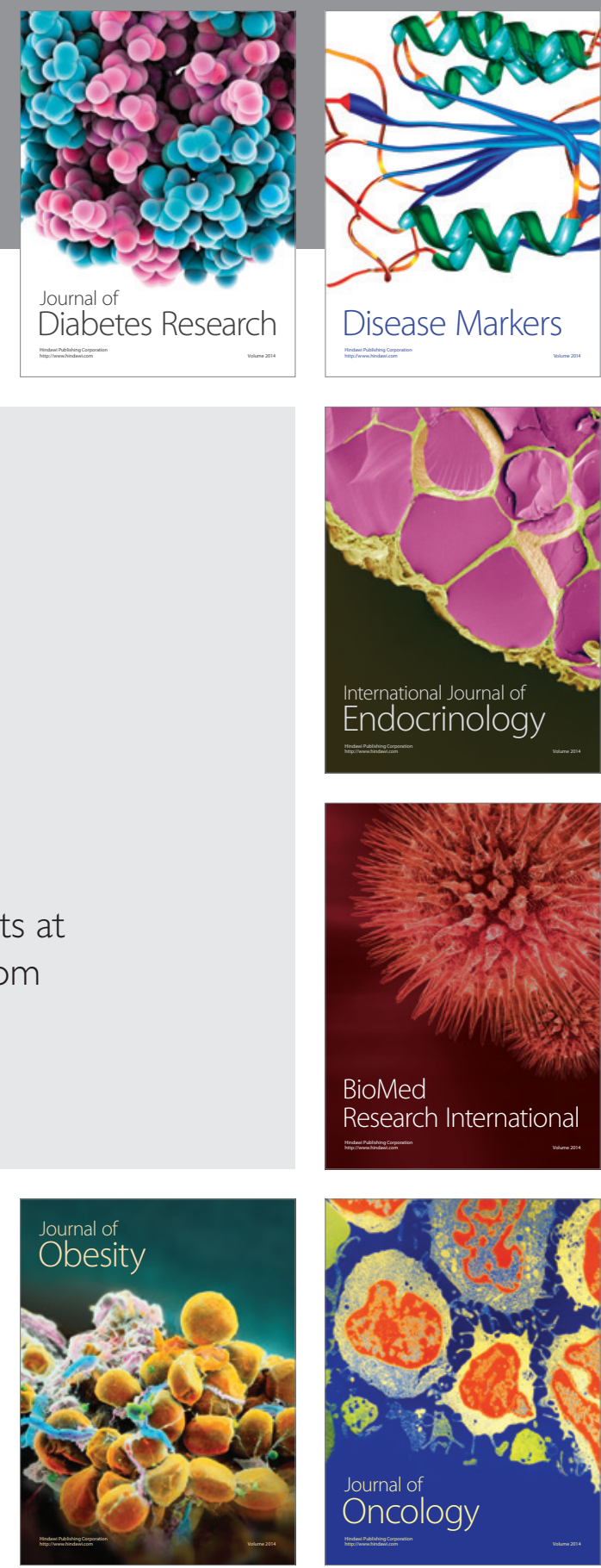

Disease Markers
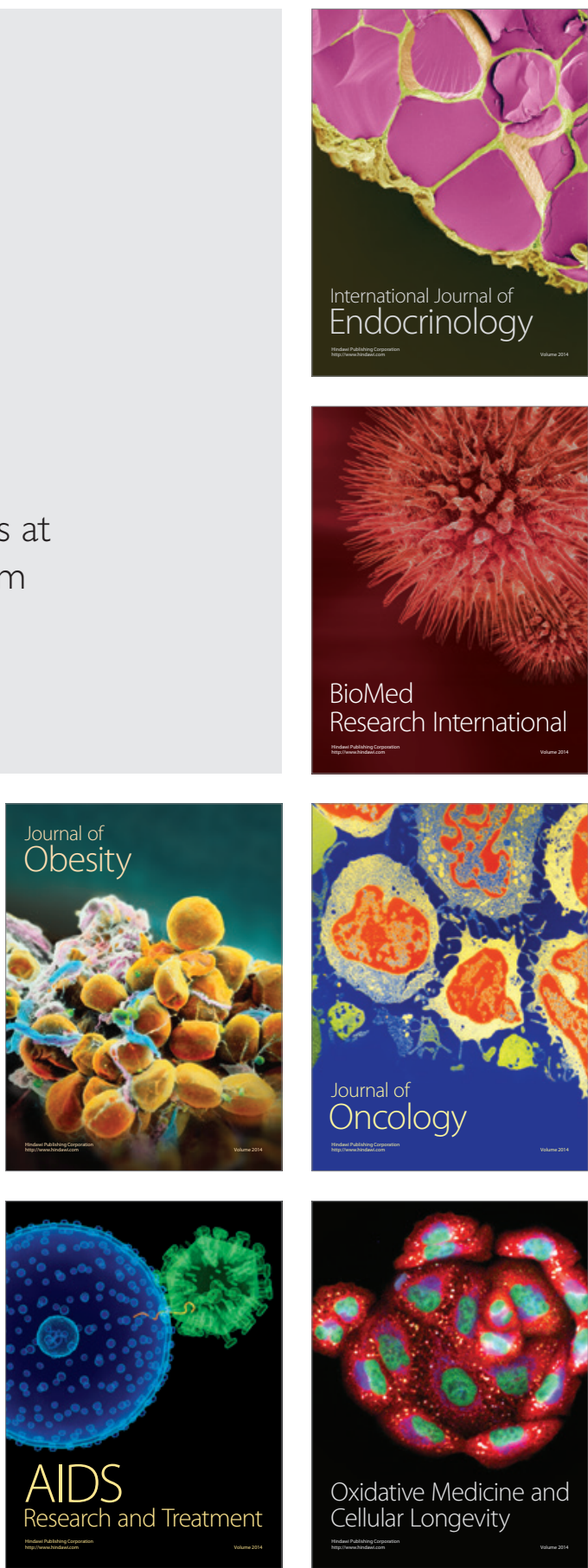